\title{
Effects of bite size and duration of oral processing on retro-nasal aroma release - features contributing to meal termination
}

\author{
Rianne M. A. J. Ruijschop ${ }^{1 *}$, Nicolien Zijlstra ${ }^{2,3}$, Alexandra E. M. Boelrijk ${ }^{4}$, Annereinou Dijkstra ${ }^{1}$, \\ Maurits J. M. Burgering ${ }^{1}$, Cees de Graaf ${ }^{3}$ and Margriet S. Westerterp-Plantenga ${ }^{5}$ \\ ${ }^{1}$ NIZO Food Research, Kernhemseweg 2, PO Box 20, 6710 BA Ede, The Netherlands \\ ${ }^{2}$ Top Institute Food and Nutrition, Nieuwe Kanaal 9a, 6709 PA, Wageningen, The Netherlands \\ ${ }^{3}$ Division of Human Nutrition, Wageningen University, Bomenweg 2, PO Box 8129, 6700 EV, Wageningen, The Netherlands \\ ${ }^{4}$ Danone Research Medical Nutrition, Bosrandweg 20, PO Box 7005, 6700 CA, Wageningen, The Netherlands \\ ${ }^{5}$ Department of Human Biology, Maastricht University, Universiteitssingel 50, PO Box 616, 6200 MD, Maastricht, \\ The Netherlands
}

(Received 25 January 2010 - Revised 20 July 2010 - Accepted 22 July 2010 - First published online 14 September 2010)

\section{Abstract}

The brain response to a retro-nasally sensed food odour signals the perception of food and it is suggested to be related to satiation. It is hypothesised that consuming food either in multiple small bite sizes or with a longer durations of oral processing may evoke substantial oral processing per gram consumed and an increase in transit time in the oral cavity. This is expected to result in a higher cumulative retronasal aroma stimulation, which in turn may lead to increased feelings of satiation and decreased food intake. Using real-time atmospheric pressure chemical ionisation-MS, in vivo retro-nasal aroma release was assessed for twenty-one young, healthy and normal-weight subjects consuming dark chocolate-flavoured custard. Subjects were exposed to both free or fixed bite size ( 5 and $15 \mathrm{~g})$ and durations of oral processing before swallowing ( 3 and $9 \mathrm{~s}$ ) in a cross-over design. For a fixed amount of dark chocolate-flavoured custard, consumption in multiple small bite sizes resulted in a significantly higher cumulative extent of retro-nasal aroma release per gram consumed compared with a smaller amount of large bite sizes. In addition, a longer duration of oral processing tended to result in a higher cumulative extent of retro-nasal aroma release per gram consumed compared with a short duration of oral processing. An interaction effect of bite size and duration of oral processing was not observed. In conclusion, decreasing bite size or increasing duration of oral processing led to a higher cumulative retro-nasal aroma stimulation per gram consumed. Hence, adapting bite size or duration of oral processing indicates that meal termination can be accelerated by increasing the extent of retro-nasal aroma release and, subsequently, the satiation.

Key words: Atmospheric pressure chemical ionisation-MS: MS-Nose: Retro-nasal aroma stimulation: Flavour: Satiation: Energy intake

During the consumption of a meal, aroma molecules reach the olfactory epithelium retro-nasally (perceived as arising from the mouth) ${ }^{(1,2)}$. The brain response, i.e. neural brain activation, to a retro-nasally sensed food odour signals the perception of food and is suggested to be related to satiation $^{(3)}$. The extent of retro-nasal aroma release during consumption depends on both product (i.e. the physical structure of a food, which has an influence on the breakdown and oral processing in the mouth ${ }^{(4-7)}$ ) and interpersonal differences ${ }^{(8)}$. Interpersonal differences are factors that are likely to be uncontrolled by a person, e.g. saliva production, nasal anatomy and oral processing habits $^{(9-13)}$. In addition to product and (uncontrolled) interpersonal features, bite size and duration of oral processing may also be of importance for the extent of retro-nasal aroma release.

Recently, Zijlstra et al. ${ }^{(14,15)}$ investigated the effect of bite size (i.e. either free or fixed ( 5 and $15 \mathrm{~g}$ )) and duration of oral processing (i.e. either free or fixed ( 3 and $9 \mathrm{~s}$ )) for a semi-liquid food product (i.e. dark chocolate-flavoured custard) on satiation/ad libitum food intake. In the fixed eating conditions, subjects consumed significantly less when bite sizes were small $(5 \mathrm{~g}$ for both short and longer duration of oral processing) than when bite sizes were

\footnotetext{
Abbreviations: ApcI, atmospheric pressure chemical ionisation; A.U., arbitrary units; AUC, area under curve; $I_{\text {max }}$, maximum intensity; $T_{\text {max }}$, time at which maximal intensity occurs; $T_{+1 / 2}$, time at which half of the maximal intensity occurs, after reaching $T_{\text {max }} ; T_{-1 / 2}$, time at which half of the maximal intensity occurs, before reaching $T_{\max }$.
} 
large ( $15 \mathrm{~g}$ for both short and longer duration of oral processing (bite size effect $P<0.05$ ) and when duration of oral processing was $9 \mathrm{~s}$ compared with $3 \mathrm{~s}$ (oral processing time effect $P<0.05)$. Ad libitum consumption in the free eating condition (without any chewing protocol) was comparable to the ad libitum consumption with large bite sizes. According to Zijlstra et al. ${ }^{(15)}$, the lower ad libitum food intake when consuming the custard in small bite sizes or with a longer duration of oral processing was probably explained by a longer oro-sensory exposure from the dark chocolate-flavoured custard to the sensory tactile and taste receptors in the oral cavity ${ }^{(16)}$. Longer oro-sensory exposure may accelerate sensory satiation, which in turn may lead to earlier meal termination ${ }^{(17,18)}$.

In this context, differences in the extent of retro-nasal aroma release may also be (partly) responsible for this effect, stimulating olfactory receptors. We hypothesise that differences in the extent of retro-nasal aroma release during consumption may be one of the reasons that people vary in their satiation characteristics, due to differences in perceived intensity, duration or quality of retronasal aroma stimulation ${ }^{(19)}$. In a recent study, we were able to show that a retro-nasally delivered aroma stimulus was capable of inducing satiation ${ }^{(20)}$. Fig. 1 illustrates some of the features that are suggested to contributing to the extent of retro-nasal aroma stimulation and, subsequently, sensory satiation, from both a person and food product point of view ${ }^{(21)}$.

Although the extent of retro-nasal aroma release appears to be subject specific, food product properties can be tailored in such a way that these can lead to a higher quality and/or quantity of retro-nasal aroma stimulation. This in turn provokes enhanced feelings of satiation and, ultimately, may contribute to a decrease in food intake.

The aim of the present study was, therefore, to provide an additional, possibly complementary, explanation for the ad libitum intake results obtained by Zijlstra et al. ${ }^{(15)}$ from a retro-nasal aroma release perspective. In addition, the results from the free eating condition served to determine whether interpersonal differences in the extent of retro-nasal aroma release can be linked to interpersonal differences in ad libitum food intake, as measured by Zijlstra et al. $^{(15)}$ and as previously reported ${ }^{(8)}$.

\section{Subjects and methods}

\section{Subjects}

Twenty-one healthy subjects (nine men and twelve women) aged 27 (SD 9) years living in Ede, the Netherlands, and surroundings participated in the present study. Initially, subjects were recruited from the subject population who participated in the study of Zijlstra et al. ${ }^{(14,15)}$. To prevent a lack of statistical power, nine subjects were recruited in-house. Subjects did not have any previous experience with atmospheric pressure chemical ionisation-MS (APcI-MS) measurements. The subjects were of normal weight, with a BMI varying between 19 and $25 \mathrm{~kg} / \mathrm{m}^{2}$. BMI was calculated as body weight ( $\mathrm{kg}$ ) divided by height $(\mathrm{m})$ squared. The present study was conducted according to the guidelines laid down in the Declaration of Helsinki and all procedures involving human subjects were approved by the ethics committee of Wageningen University. Subjects were fully informed about the course of the APCI-MS measurement and they gave their written, informed consent. To prevent response-bias, the participants

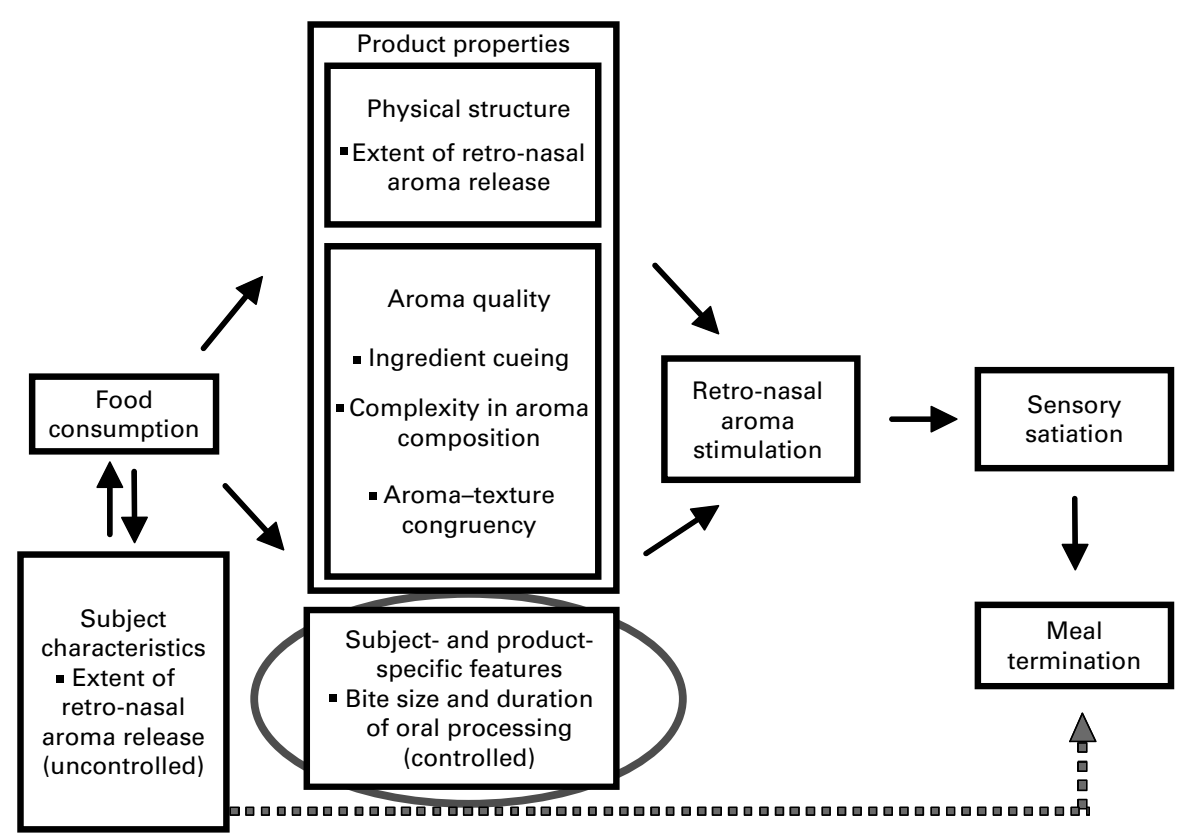

Fig. 1. Schematic representation of features that are suggested to contributing to the extent of retro-nasal aroma stimulation and sensory satiation, from both consumer and food product points of view (adapted from Ruijschop et al. ${ }^{(21)}$ ). The topic of the present study is encircled. 
were given no information about the hypothesis and nature of the predictions of the experiment. They were only aware of the fact that the study was about in vivo retro-nasal aroma release assessment.

\section{Food product}

Subjects consumed dark chocolate-flavoured custard (Royal FrieslandCampina, Deventer, the Netherlands). The physical structure of the custard can be characterised as semi-liquid with a viscosity of 43 (SD 11) s, as measured with a Posthumus funnel.

To check for a clear aroma signal in the APcI-MS measurement, the dark chocolate-flavoured custard was spiked with methyl acetate. Methyl acetate (Sigma-Aldrich Chemie GmbH, Steinheim, Germany) was applied as a volatile marker aroma molecule, which was rapidly and easily released from the food product matrix during consumption. The sensory quality of methyl acetate can be described as fruity and sweet. In finished consumer products, methyl acetate concentrations vary from $0 \cdot 1$ to $30 \mathrm{mg} / \mathrm{kg}^{(22)}$. Methyl acetate solution $(0 \cdot 1 \mathrm{ml})$ with a concentration of $100 \mathrm{~g} / \mathrm{kg}$, i.e. diluted ten fold in propylene glycol (private label from a local pharmacy in Ede, the Netherlands), was added to 1 litre dark chocolateflavoured custard following a standardised procedure of ten times gently stirring. The methyl acetate concentration in the dark chocolate-flavoured custard was $10 \mathrm{mg} / \mathrm{kg}$. The dark chocolate-flavoured custard product was left overnight at $4^{\circ} \mathrm{C}$ to allow a good equilibration of the aroma in the product.

The viscosity of the dark chocolate-flavoured custard product was not affected by the addition of methyl acetate. Moreover, there were no sensory differences perceived between the dark chocolate-flavoured custards with or without addition of methyl acetate $(P=0 \cdot 12)$. This was evaluated by an in-house trained sensory panel (eleven panellists), who performed a triangle test.

Measurement of the extent of retro-nasal aroma release with atmospheric pressure chemical ionisation-MS technology

To detect the aroma compounds with the highest response in retro-nasal aroma release, the dynamic headspace of the dark chocolate-flavoured custard product was measured. Aroma compounds in the air released from the artificial mouth $^{(23)}$ were monitored by online sampling by an APcI-gas phase analyser attached to a VG Quattro II mass spectrometer (MS-Nose; Micromass UK Limited, Manchester, UK $)^{(24-27)}$. Compounds were ionised by a $3.0 \mathrm{kV}$ discharge (source and probe temperature were $80^{\circ} \mathrm{C}$ ) and scanned for $m / z 50-250 . m / z$ Values with the highest response were selected, i.e. $\mathrm{m} / z$ 69, 75 and 87 . Both $\mathrm{m} / z$ 69 and 87 were originally present in the dark chocolateflavoured custard, while $m / z 75$ represented the marker aroma molecule, methyl acetate. In vivo retro-nasal aroma release was assessed in exhaled breath of the subjects. Subjects breathed in and out through the nose. One nostril was placed over a small disposable plastic tube, allowing them to breathe and eat normally. Aroma compounds in the air released from the breath of subjects were monitored by online sampling of part of the exhaled air directly into the APcI-MS. The air was sampled $(75 \mathrm{ml} / \mathrm{min})$ through a capillary tube $(0.53 \mathrm{~mm}$ internal diameter, heated to $100^{\circ} \mathrm{C}$ ). The compounds were monitored in selected ion mode $(0.08 \mathrm{~s}$ dwell on each ion). The cone voltage used was $20 \mathrm{~V}$.

Acetone, present in human breath, was measured at $\mathrm{m} / \mathrm{z}$ $59(19 \mathrm{~V})$ as an indicator of the breathing pattern ${ }^{(28)}$. The area of the resulting breath peaks in the aroma signal was taken as a measure of in vivo retro-nasal aroma release. Different parameters could be extracted from each individual retro-nasal aroma release curve, characterising the extent of retro-nasal aroma release, i.e. time at which maximal intensity occurs $\left(T_{\max }\right)$, time at which half of the maximal intensity occurs, before reaching $T_{\max }$ $\left(T_{-1 / 2}\right)$, time at which half of the maximal intensity occurs, after reaching $T_{\max }\left(T_{+1 / 2}\right)$, maximum intensity $\left(I_{\max }\right)$ and area under curve (AUC) (Fig. 2). Since we were interested in comparative retro-nasal aroma release between subjects, expression of the extent of retro-nasal aroma release in arbitrary units (A.U.) was sufficient to analyse the differences ${ }^{(24)}$.

\section{Experimental design}

In vivo retro-nasal aroma release was assessed in exhaled breath of the twenty-one subjects for the dark chocolateflavoured custard product in quintuplicate according to a specific protocol established to determine the effect of bite size and oral processing time before swallowing on the extent of retro-nasal aroma release ${ }^{(14,15)}$. To this end, subjects were exposed to five different eating conditions

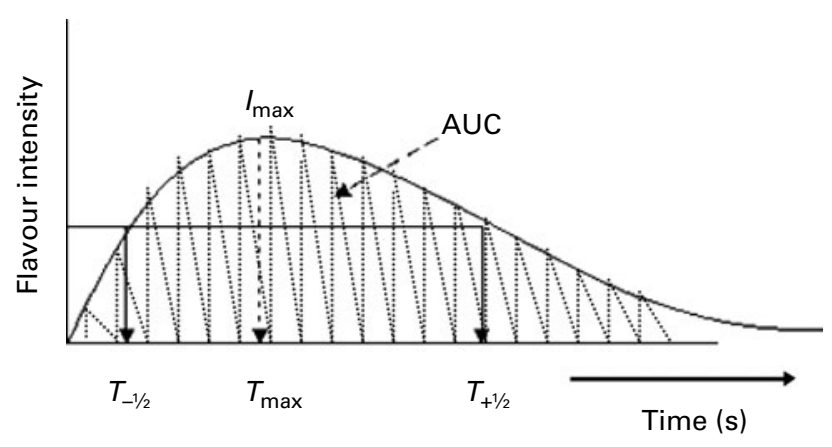

Fig. 2. Schematic representation of a retro-nasal aroma release curve and its characteristic parameters. $I_{\max }$, Maximum intensity (arbitrary units (A.U.)); $T_{\max }$, time at which maximal intensity occurs (min); AUC, total area under the curve (A.U.); $T_{-1 / 2}$, time at which half of the maximal intensity occurs, before reaching $T_{\max }(\min ) ; T_{+1 / 2}$, time at which half of the maximal intensity occurs, after reaching $\left.T_{\max }(\min )\right)$. 


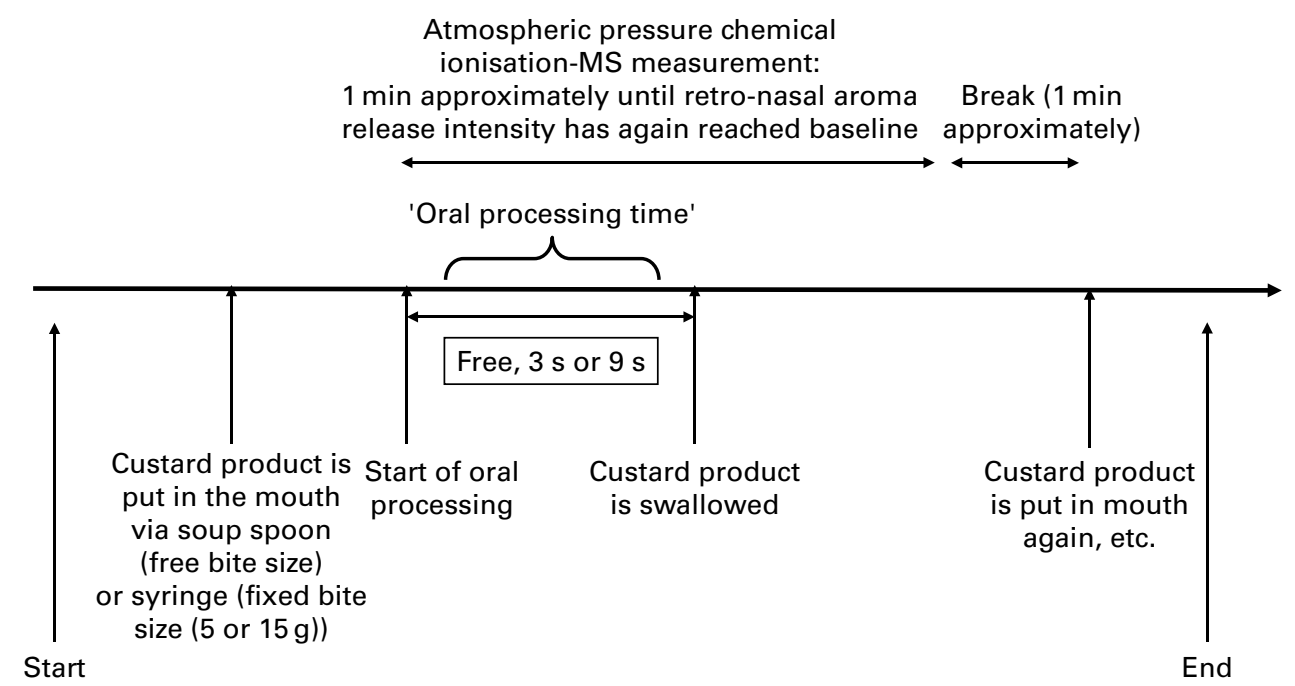

Fig. 3. Overview of the protocol for in vivo retro-nasal aroma release assessment in either the free or the different fixed eating conditions for the dark chocolateflavoured custard product.

in a cross-over design, in which bite size was either small $(5 \mathrm{~g})$ or large $(15 \mathrm{~g})$, and oral processing time before swallowing was either short (3s) or long (9s), or subjects were free to use their own natural eating habits, including free bite size and free oral processing time before swallowing. Treatment order for the fixed eating conditions was counterbalanced across both test days and subjects. In the free eating condition, subjects consumed five times one mouthful of dark chocolate-flavoured custard product by means of a soup spoon without any chewing protocol. It has to be noticed that the size of the soup spoon may have affected the bite size in the free eating condition. In the fixed eating conditions, subjects were instructed to put the dark chocolate-flavoured custard product (either 5 or $15 \mathrm{~g}$, presented in a syringe) in the mouth, to close the mouth and to actively move the custard product in the mouth with the tongue for either 3 or $9 \mathrm{~s}$, thereby simulating oral processing in the mouth, and then to swallow the entire bolus. After consumption, the syringe was weighed to determine the precise bite size of custard product per serving (Mettler-Toledo balance, Greifensee, Switzerland).

Between the different eating conditions, the mouth was rinsed with water. Blank experiments with water were recorded before consuming the dark chocolate-flavoured custard product in the different eating conditions, following the same protocol as for the first fixed eating condition. These experiments served as a baseline measurement. The protocol for either the free or the fixed eating conditions is represented schematically in Fig. 3. During the measurements, the subjects were closely observed in order to verify their compliance with the protocol. Fig. 4 illustrates a subject's retro-nasal aroma release curve for the different eating conditions.

\section{Measurement of ad libitum food intake}

For a subset of twelve subjects (five men and seven women; aged 21 (SD 3) years; BMI 22 (SD 1) kg/m²), showing no dietary restraint (i.e. men: score $\leq 2 \cdot 89$, women: score $\leq 3.40$ on Dutch Eating Behaviour Questionnaire), ad libitum food intake data of the dark chocolateflavoured custard product was available from a study of Zijlstra et al. ${ }^{(14,15)}$. In the present study, the effect of bite size and oral processing time for dark chocolate-flavoured custard on satiation/ad libitum food intake was determined. To assess whether interpersonal differences in the extent of retro-nasal aroma release can be linked to interpersonal differences in ad libitum food intake, the amount of dark chocolate-flavoured custard consumed ad libitum in the free eating condition in the satiation experiment performed by Zijlstra et al. ${ }^{(15)}$ was related to the extent of retro-nasal aroma release per gram consumed for these subjects in the free eating condition.

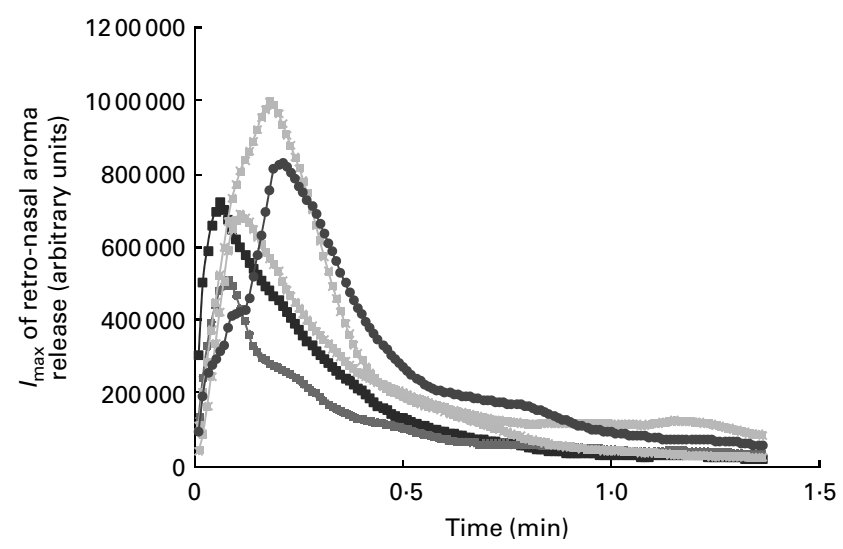

Fig. 4. Illustration of a subject's retro-nasal aroma release curve for the different eating conditions. As depicted, differences in oral processing time before swallowing (short $v$. long) significantly affect $T_{\max }$ for both small and large bite sizes. $\longrightarrow$, Free, free; $\longrightarrow$, small, short; - , small, long; $\because$, large, short; $\multimap$, large, long. 


\section{Data analysis}

The quintuplicate measurements of the characteristic retronasal aroma release parameters $\left(T_{\max }, I_{\max }\right.$ and AUC) for each subject per eating condition were averaged. This is allowed, since subjects are reproducible in their extent of retro-nasal aroma release during consumption of a specific food product ${ }^{(8,10)}$. Due to the lower signal-to-noise ratio for the custard-specific $\mathrm{m} / z$ values 69 and 87 compared with the marker aroma molecule with $m / z$ value 75 , the background noise, as measured with the blank experiments, was subtracted from the measurements with the dark chocolate-flavoured custard product in the different eating conditions per subject for all $\mathrm{m} / z$ values.

All data are presented as means with their standard errors. The different characteristic retro-nasal aroma release parameters were compared between the five different eating conditions per bite size or per gram consumed using the general linear model procedure for repeated measures, with participant and type of eating condition as independent variables. Least squares means were used for post hoc comparisons. Multiple regression analysis $(f(x))$ was performed for the characteristic retro-nasal aroma release parameters $(y)$, and bite size $(x)$ and oral processing time before swallowing $(x)$.

Additionally, the extent of retro-nasal aroma release $(y)$ was linked to subject characteristics, such as age $(x)$ and BMI $(x)$, and ad libitum food intake $(x)$, applying simple regression analysis $(f(x))$. For all the data analyses, the statistical packages Statistical Analysis Systems (release 9.1; SAS Institute, Inc., Cary, NC, USA) were used. $P$-values $<0.05$ were considered statistically significant.

\section{Results}

Bite size differences affecting the extent of retro-nasal aroma release

Bite size was 13.6 (SD 0.4) g per mouthful (range 6.7-18.2 g) in the free eating condition.

\section{Consumption of a small v. large bite size}

In the fixed eating conditions, subjects consumed 5.7 (SD 0.4) and 15.7 (SD 0.4) $\mathrm{g}$ for the small and large bite size, respectively. Subjects differed in the extent of retro-nasal aroma release regarding intensity $\left(I_{\max }\right)$ and AUC of the retronasal aroma release profile during the consumption of either small or large bite sizes of dark chocolate-flavoured custard product in the fixed eating conditions $(F(1,41)=16 \cdot 88, P<0.01$ and $F(1,41)=27 \cdot 50, P<0.01$ for $I_{\max }$ and AUC, respectively). As illustrated in Fig. 5, small bite sizes resulted in a significantly less intense retro-nasal aroma release characteristic for dark chocolate-flavoured custard product (Fig. 5(a); mean $I_{\max }$ (means with their standard errors): $3 \cdot 62 \mathrm{E}+05 \pm 2 \cdot 77 \mathrm{E}+04$ A.U. and Fig. $5(\mathrm{~b})$; mean AUC (means with their standard errors): $2.51 \mathrm{E}$
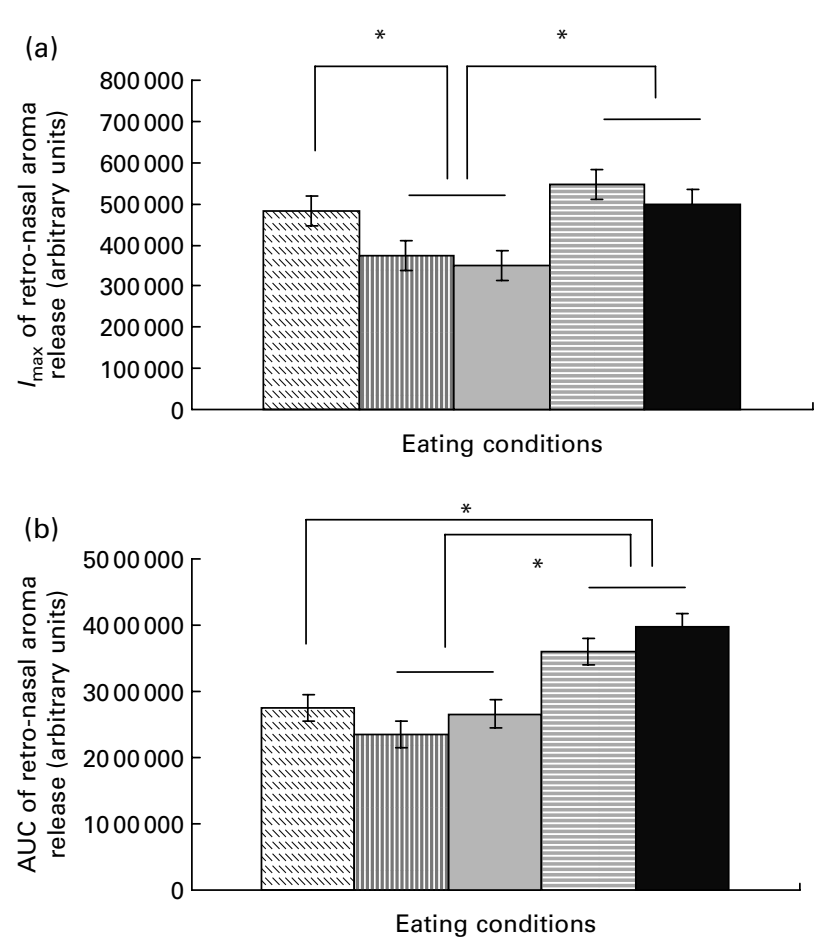

Fig. 5. Illustration of the differences in retro-nasal aroma release intensity $\left(I_{\max }(\mathrm{a})\right.$ and AUC (b)) between the consumption of dark chocolate-flavoured custard product in either the free (free bite size and free oral processing time before swallowing (free, free (国)) or the different fixed (small bite size and short oral processing time (small, short $(\mathbb{W l})$ ); small bite size and long oral processing time (small, long $(\square)$ ); large bite size and short oral processing time (large, short $(\boxminus)$ ); and large bite size and long oral processing time (large, long $(\boldsymbol{\square})$ ) eating conditions. Mean values with their standard errors are depicted by vertical bars. * Mean values denote the effect of bite size on retro-nasal aroma release intensity with $P<0.05$.

$+06 \pm 1.73 \mathrm{E}+05$ A.U.) compared with large bite sizes (Fig. 5(a); mean $I_{\max }$ (means with their standard errors): $5 \cdot 23 \mathrm{E}+05 \pm 2 \cdot 77 \mathrm{E}+04$ A.U., and Fig. 5(b); mean AUC (means with their standard errors): $3 \cdot 79 \mathrm{E}+06 \pm 1 \cdot 73 \mathrm{E}$ +05 A.U.). Bite size in the free eating condition showed comparable retro-nasal aroma release characteristics to small bite sizes for AUC and to large bite sizes for $I_{\max }$ in the fixed eating conditions (Fig. 5(a); mean $I_{\max }$ (means with their standard errors): $4.82 \mathrm{E}+05 \pm 3 \cdot 54 \mathrm{E}+04$ A.U., and Fig. 5(b); mean AUC (means with their standard errors): $2 \cdot 75 \mathrm{E}+06 \pm 2 \cdot 07 \mathrm{E}+05$ A.U.).

\section{Consumption of a fixed amount in a small or large bite size}

By expressing the effect of bite size on the extent of retronasal aroma release per gram consumed, a different view of the results was obtained. As shown in Fig. 6, small bite sizes resulted in a significantly more intense retronasal aroma release characteristic for dark chocolateflavoured custard product per gram consumed (Fig. 6(a); mean $I_{\max }$ (means with their standard errors): $6 \cdot 34 \mathrm{E}+04$ $\pm 3 \cdot 10 \mathrm{E}+03$ A.U./g consumed, and Fig. 6(b); mean AUC (means with their standard errors): $4 \cdot 38 \mathrm{E}+05 \pm 1 \cdot 77 \mathrm{E}$ +04 A.U./g consumed) compared with large bite sizes (Fig. 6(a); mean $\mathrm{I}_{\max }$ (means with their standard errors): 
$3 \cdot 33 \mathrm{E}+04 \pm 3 \cdot 10 \mathrm{E}+03$ A.U./g consumed, and Fig. 6(b); mean AUC (means with their standard errors): $2 \cdot 40 \mathrm{E}+05 \pm 1 \cdot 77 \mathrm{E}+04$ A.U./g consumed $)(F(1,41)=47 \cdot 33$ $P<0.01$ and $F(1,41)=62.01, P<0.01$ for $I_{\max }$ and AUC, respectively). There is almost a difference by a factor of two in retro-nasal aroma release $I_{\max }$ and AUC per gram consumed between the small and large bite sizes. Bite size in the free eating condition showed comparable retro-nasal aroma release characteristics per gram consumed to the large bite sizes in the fixed eating conditions (Fig. 6(a); mean $I_{\max }$ (means with their standard errors): $4 \cdot 12 \mathrm{E}+04 \pm 4 \cdot 56 \mathrm{E}+03$ A.U./g consumed, and Fig. 6(b); mean AUC (means with their standard errors): $2 \cdot 42 \mathrm{E}+05 \pm 2.58 \mathrm{E}+04$ A.U./g consumed). When the subjects were free to use their own natural eating habits, they consumed large bite sizes according to their extent of retro-nasal aroma release per gram consumed.

\section{Oral processing time differences affecting the extent of retro-nasal aroma release}

The morphology of the retro-nasal aroma release profile (in particular explained by $T_{\max }$, i.e. time at which $I_{\max }$
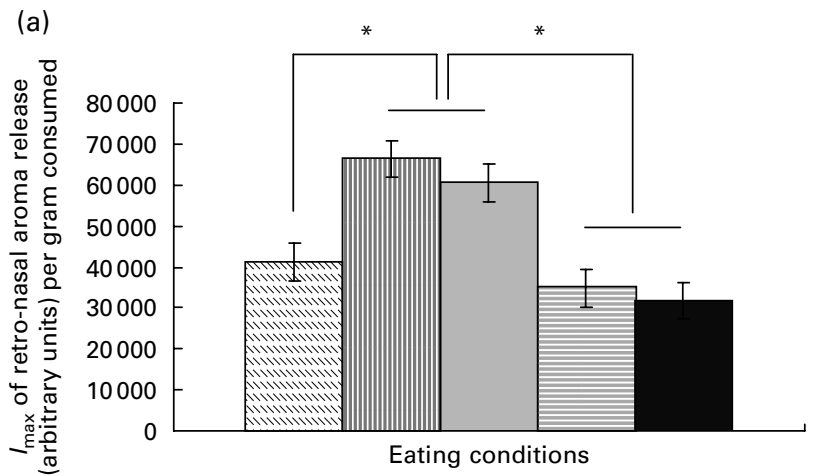

(b)
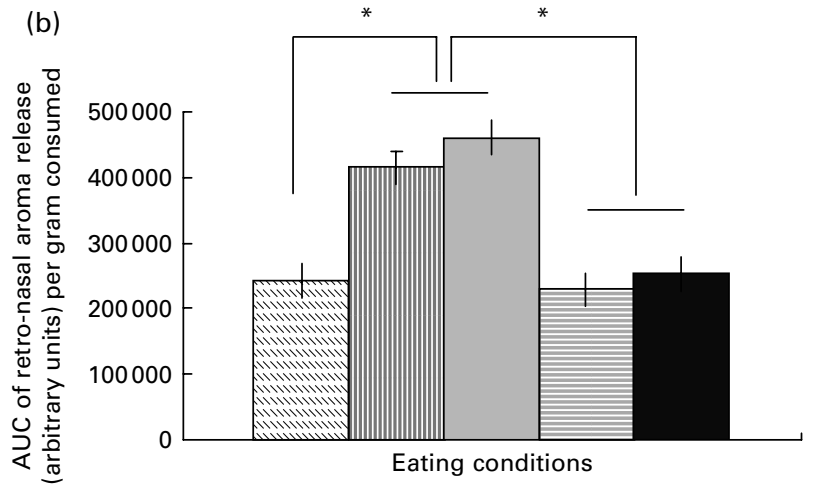

Fig. 6. Illustration of the differences in retro-nasal aroma release intensity $\left(I_{\max }(\mathrm{a})\right.$ and area under curve $\left.(\mathrm{b})\right)$ between the consumption of dark chocolate-flavoured custard product per gram consumed in either the free (free bite size and free oral processing time before swallowing (free, free (因)) or the different fixed (small bite size and short oral processing time (small, short $(\mathbb{W})$ ); small bite size and long oral processing time (small, long ( $\square$ )); large bite size and short oral processing time (large, short $(\boxminus)$ ); and large bite size and long oral processing time (large, long $(\boldsymbol{\square})$ ) eating conditions. Mean values with their standard errors are depicted by vertical bars. * Mean values denote the effect of bite size on retro-nasal aroma release intensity with $P<0.05$.

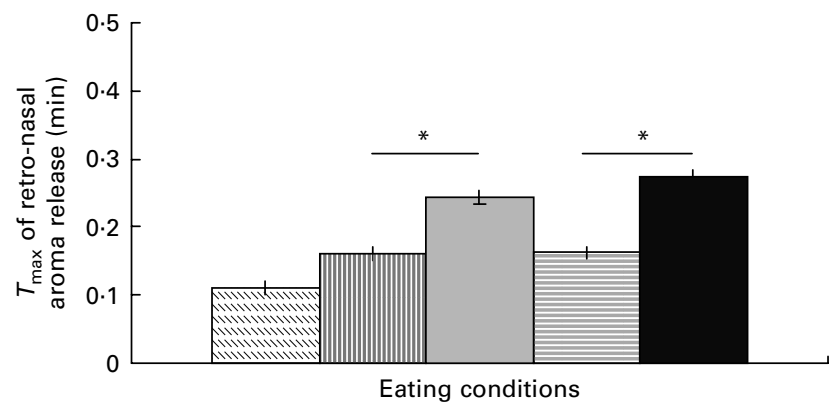

Fig. 7. Morphology of the retro-nasal aroma release profile for the dark chocolate-flavoured custard product in the different eating conditions. Free, free (因); small, short (四); small, long ( $\square$ ); large, short (目) and large, long (ם). There is a distinct difference in duration of oral processing for a fixed bite size in the different fixed eating conditions for $T_{\max }$ (i.e. time at which maximal intensity occurs (min)). Mean values with their standard errors are depicted by vertical bars. * Mean values denote the effect of duration of oral processing on $T_{\max }$ with $P<0.05$.

occurred) was different between the short (3s) and longer (9s) duration of oral processing for a fixed bite size in the different fixed eating conditions (Fig. 7; mean $T_{\max }$ (means with their standard errors) for the small bite size: $0 \cdot 16$ (SEM 0.01) and 0.24 (SEM 0.01) min for the short and longer duration of oral processing, respectively; and mean $T_{\max }$ (means with their standard errors) for the large bite size: $0 \cdot 16$ (SEM $0 \cdot 01$ ) and $0 \cdot 27$ (SEM 0.01) min for the short and longer duration of oral processing, respectively). In compliance with the protocol, subjects swallowed later when they were exposed to the fixed eating condition of a given bite size and a longer duration of oral processing. Hence, a significant delay in $T_{\max }$ was observed compared with the short duration of oral proces$\operatorname{sing}(P<0 \cdot 01)$. In the free eating condition, $T_{\max }$ was $0 \cdot 11$ (sem $0 \cdot 01) \mathrm{min}$ (range $0 \cdot 05-0 \cdot 24 \mathrm{~min}$ ). Although significantly different from the fixed eating conditions $(P<0.01), T_{\max }$ in the free eating condition approximated the short duration of oral processing more than the longer duration of oral processing.

\section{Short v. long duration of oral processing during consumption of different bite sizes}

Apart from differences in bite size affecting the extent of retro-nasal aroma release, differences in duration of oral processing time were also thought to be important for the extent of retro-nasal aroma release. A longer duration of oral processing resulted in a significantly higher extent of retro-nasal aroma release (AUC) for dark chocolateflavoured custard product in the fixed eating conditions (Fig. 5(b); $2 \cdot 98 \mathrm{E}+06 \pm 1 \cdot 13 \mathrm{E}+05$ A.U. and $3 \cdot 31 \mathrm{E}+06$ $\pm 1 \cdot 13 \mathrm{E}+05$ A.U. for the short and longer duration of oral processing, respectively) $(F(1,41)=4 \cdot 40, \quad P=0 \cdot 04)$. The duration of oral processing in the free eating condition showed comparable retro-nasal aroma release characteristics to small bite sizes with both short and longer duration of oral processing for AUC and to large bite sizes with both short and longer duration of oral processing for $I_{\max }$ in the fixed eating conditions. 
Short v. long duration of oral processing during consumption of a fixed amount

During the consumption of a fixed amount of dark chocolate-flavoured custard product, a longer duration of oral processing tended to result in a higher cumulative extent of retro-nasal aroma release (AUC) for dark chocolate-flavoured custard product per gram consumed in the fixed eating conditions (Fig. 6(b); 3.22E +05 $\pm 1 \cdot 40 \mathrm{E}+04$ A.U./g consumed and $3 \cdot 57 \mathrm{E}+05 \pm 1 \cdot 40 \mathrm{E}$ +04 A.U./g consumed for the short and longer duration of oral processing, respectively) $(F(1,41)=3 \cdot 16, P=0 \cdot 08)$. The duration of oral processing in the free eating condition showed comparable retro-nasal aroma release characteristics (AUC and $I_{\max }$ ) per gram consumed to both short and longer duration of oral processing of large bite sizes. The differences in bite size affected the extent of retro-nasal aroma release per gram consumed significantly compared with the difference in duration of oral processing in the fixed eating conditions $(y=-1.92 \mathrm{E}$ $+04 x+5.44 \mathrm{E}+05, \quad R^{2} \quad 0.19, \quad P<0.01$ and $y=5.85 \mathrm{E}$ $+03 x+3 \cdot 04 \mathrm{E}+05, R^{2} 0 \cdot 01, P=0.47$ for the effect of bite size $(x)$ and duration of oral processing $(x)$ on the extent of retro-nasal aroma release per gram consumed (AUC; $y$ ), respectively). This indicates that the bite size effect presumably overwhelmed the effect of duration of oral processing. Therefore, it is likely that in the free eating condition, bite size accounted for the present result rather than the duration of oral processing.

\section{Discussion}

Using APcI-MS technology, in vivo retro-nasal aroma release was assessed for twenty-one subjects consuming dark chocolate-flavoured custard in either a free or a fixed eating condition.

Selected aroma compounds detected in the range of $\mathrm{m} / \mathrm{z}$ $20-250$, i.e. aroma compounds with the highest response, showed comparable retro-nasal aroma release profiles for the dark chocolate-flavoured custard product. From previous studies, it is known that aroma release data obtained for a single aroma compound (one specific $m / z$ value) are a good predictor for the relative release of other aroma compounds (other $m / z$ values) ${ }^{(8)}$. Therefore, the same relative retro-nasal aroma release profile was measured, irrespective of the $m / z$ value taken. However, it appeared that the signal-to-noise ratio for the custard-specific $\mathrm{m} / z$ values of 69 and 87 was lower compared with the marker aroma molecule with $m / z 75$. The custard-specific $m / z$ values provided a less clear aroma signal in the APcI-MS measurements compared with the marker $\mathrm{m} / \mathrm{z}$ value. Although the sensory detection thresholds of both custard-specific aroma molecules were probably low enough to be detected by a human nose via the retro-nasal route, the 'MS-Nose' might be less sensitive in detecting the custard-specific aroma molecules in a concentration as applied in dark chocolate-flavoured custard. After baseline correction, $m / z 75$ had the highest response for the dark chocolate-flavoured custard product and was selected for the in vivo retro-nasal aroma release measurements.

The extent of retro-nasal aroma release from small bite sizes of dark chocolate-flavoured custard product was significantly lower compared with large bite sizes (Fig. 5). However, for a fixed amount of dark chocolate-flavoured custard product, consumption in numerous small bite sizes resulted in a significantly higher cumulative extent of retro-nasal aroma release per gram consumed compared with a smaller number of, but larger, bite sizes (Fig. 6) These bite size effects were in line with our expectations.

When subjects consumed large bite sizes, they were probably exposed to higher quantities of aroma molecules, because in absolute value, a larger bite size contains more aroma molecules than a smaller bite size. However, it should be mentioned that from the total amount of aroma molecules present in food, only a small fraction of the aroma is released into the nasal cavity during oral processing and swallowing. The majority of aroma molecules is swallowed together with the food matrix into the stomach and is no longer available for retro-nasal aroma perception $^{(29)}$. Besides, large bite sizes presumably led to more frequent swallowing. Retro-nasal aroma stimulation is mainly related to the event of swallowing, when a small volume of air is exhaled immediately after swallowing, the so-called 'swallow-breath'. It is assumed that this pulse should contain the major part of food volatiles that have been released from the food product before swallowing and should therefore elicit a retro-nasal aroma pulse ${ }^{(29,30)}$. Large bite sizes thus yielded a higher extent of retro-nasal aroma release; but per larger bite also led to a higher food intake compared with small bite sizes.

Alternatively, for the same amount of food intake, subjects had a higher cumulative extent of retro-nasal aroma release per gram if they consumed the custard in a multiple of small bite sizes compared with a smaller number of large bite sizes (Fig. 6). For example, when subjects consumed a fixed amount of $15 \mathrm{~g}$ of dark chocolate-flavoured custard product in either one large or three small bite sizes, the multiple small bite sizes were hypothesised to evoke substantial oral processing per gram consumed and an increase in transit time in the oral cavity ${ }^{(8)}$. Accordingly, the three multiple small bite sizes of $5 \mathrm{~g}$ of dark chocolate-flavoured custard product were expected to lead to a higher cumulative retro-nasal aroma stimulation compared with the single large bite size of $15 \mathrm{~g}$.

In addition to the bite size effect, a longer duration of oral processing before swallowing tended to result in a higher cumulative extent of retro-nasal aroma release per gram consumed compared with a short duration of oral processing before swallowing (Fig. 6). A longer duration of oral processing was thought to evoke an increase in transit time in the oral cavity and thereby result in a higher extent of retro-nasal aroma release. Besides, during a longer 
duration of oral processing, the temperature of the dark chocolate-flavoured custard product in the mouth may further increase towards body temperature and probably more surface of the custard product is exposed to air, both resulting in a higher quantitative release of aroma molecules from the custard matrix.

The effect of oral processing time was less strong than the effect of the bite size. With dark chocolate-flavoured custard as the experimental food product, this result was not unexpected. The morphology of the retro-nasal aroma release curve for a custard product can be described as a relatively short and spiked retro-nasal aroma release pattern ${ }^{(8,26)}$. The more liquid food products have a relatively short transit time in the oral cavity and therefore hardly any oral processing is needed to swallow them. In the present study, subjects actively moved the custard product in the mouth with the tongue for either 3 or $9 \mathrm{~s}$, thereby simulating oral processing in the mouth. However, the event of swallowing was probably not affected by varying the duration of oral processing. Therefore, the difference in duration of oral processing may not evoke a significant difference in retro-nasal aroma stimulation. The observed differences in the extent of retro-nasal aroma release between the short and longer duration of oral processing are likely to be due to intermittent opening of the connection of the oral cavity to the naso- and dorsal oropharynx. During the simulation of oral processing in the mouth, the velum was assumed to be open, which enabled air to proceed into the nasal cavity via the retronasal route through the velopharyngeal portal ${ }^{(10)}$. To strengthen this physiological effect, increasing the difference in duration between the short and longer duration of oral processing may increase the amount of air which reaches the nasal cavity via the retro-nasal route during an increased longer duration of oral processing. Alternatively, exposing subjects to a fixed protocol for swallowing, meaning that they have to swallow more frequently during the longer duration of oral processing compared with the short duration of oral processing, may increase the effect of oral processing time. It may even be more efficient than a longer duration of oral processing with respect to the extent of retro-nasal aroma release. Moreover, the choice of a more solid food product as the experimental food product may strengthen the impact of a longer duration of oral processing on the extent of retro-nasal aroma release. Solid food products require considerable oral processing and swallowing, due to their firmer texture $^{(8,10)}$. Since aroma pulses are generated along with each swallow-breath, solid food products generate a longer, more pronounced retro-nasal aroma release than liquid food products ${ }^{(20)}$.

In the present study, an interaction effect of bite size and duration of oral processing was not observed.

To summarise, small bite sizes contributed significantly to a higher cumulative extent of retro-nasal aroma release per gram, whereas a longer duration of oral processing tended to result in a higher extent of retro-nasal aroma release during consumption of a fixed amount. However, the effect of oral processing time may be significant with a more solid food product.

As reported by Zijlstra et al. ${ }^{(14,15)}$, consumption in small bite sizes or with a longer duration of oral processing resulted in a lower ad libitum food intake. The results of the present study provided an additional, possibly complementary, explanation for the ad libitum intake results obtained by Zijlstra et al. ${ }^{(15)}$ from a retro-nasal aroma release perspective, stimulating olfactory receptors. Differences in the extent of retro-nasal aroma release may thus be (partly) responsible for the decrease in food intake in certain eating conditions.

When the subjects were free to use their own natural eating habits, including free bite size and free oral processing time before swallowing, the extent of retro-nasal aroma release per gram consumed reflected the extent of retro-nasal aroma release per gram consumed from large bite sizes, while duration of oral processing was more difficult to estimate. $T_{\max }$ in the free eating condition approximated the short duration of oral processing more than the longer duration of oral processing (Fig. 7). The physical structure of the custard product was probably responsible for this result. Generally, hardly any oral processing is needed under natural eating conditions to swallow (semi)-liquid food products.

In the present study, for the subset of twelve subjects, no significant relationship was observed between the extent of retro-nasal aroma release per gram consumed in the free eating condition and the amount of dark chocolateflavoured custard consumed ad libitum in the free eating condition in the satiation experiment done by Zijlstra et $a{ }^{(15)}$. Moreover, the subject characteristics, such as sex, age and BMI, did not correlate with the extent of retro-nasal aroma release per gram consumed. This result was not unexpected. The interpersonal differences in the extent of retro-nasal aroma release per gram consumed, which were hypothesised to be linked to the interpersonal differences in ad libitum food intake ${ }^{(8)}$, were likely to be too small within the subset of twelve subjects. Subjects included were rather homogeneous, i.e. normal weight and of similar age. A follow-up study with a larger subject population, which is more segmented with respect to age and BMI, is needed to demonstrate a significant effect of the extent of retro-nasal aroma release on ad libitum food intake ${ }^{(8)}$. However, this is usually conflicting with the subject requirements for satiation experiments, i.e. homogeneous.

The concept that small bite sizes or a longer duration of oral processing may lead to a higher cumulative retro-nasal aroma stimulation indicates that meal termination can be accelerated by increasing the extent of retro-nasal aroma release and, subsequently, the satiation. Bite size and duration of oral processing are features which may be subjected to behavioural changes as a result of, for instance, 
mindful eating ${ }^{(31)}$. In this context, one piece of advice from dietitians when participating in a weight loss programme is to consume small bite sizes and to chew properly (e.g. ten times) on each bite before swallowing. The development of long chewable food structures that evoke substantial oral processing and an increase in transit time in the oral cavity or a reduction in bite size by tailored packaging may be product development suggestions that support the 'right' oral processing behaviour. These applications may lead to a higher extent of retro-nasal aroma release and sensory stimulation, which in turn may affect satiation and food intake behaviour.

\section{Acknowledgements}

We acknowledge Carina Ponne, PhD (Royal FrieslandCampina), for providing the dark chocolate-flavoured custard.

R. M. A. J. R. designed the experiment, analysed the data and wrote the manuscript. N. Z. performed subject recruitment and designed the experiment. A. E. M. B. provided significant advice on the study design, data analysis and helped to prepare the manuscript. A. D. collected the data and helped to analyse the data. M. J. M. B. reviewed the manuscript. C. d. G. and M. S. W.-P. provided significant advice with the design of the experiment, data analysis and writing of the manuscript. None of the authors had a personal or financial conflict of interest.

\section{References}

1. Murphy C, Cain WS \& Bartoshuk LM (1977) Mutual action of taste and olfaction. Sens Processes 1, 204-211.

2. Rozin P (1982) Taste-smell confusions and the duality of the olfactory sense. Percept Psychophys 31, 397-401.

3. Small DM, Gerber JC, Mak YE, et al. (2005) Differential neural responses evoked by orthonasal versus retro-nasal odorant perception in humans. Neuron 47, 593-605.

4. Linforth RST, Baek I \& Taylor AJ (1999) Simultaneous instrumental and sensory analysis of volatile release from gelatine and pectin/gelatine gels. Food Chem 65, 77-83.

5. Cook DJ, Linforth RST \& Taylor A (2003) Effects on hydrocolloid thickeners on the perception of savory flavors. J Agric Food Chem 51, 3067-3072.

6. Lethuaut L, Weel KG, Boelrijk AE, et al. (2004) Flavor perception and aroma release from model dairy desserts. J Agric Food Chem 52, 3478-3485.

7. van Ruth SM, de Witte L \& Uriarte AR (2004) Volatile flavor analysis and sensory evaluation of custard desserts varying in type and concentration of carboxymethyl cellulose. J Agric Food Chem 52, 8105-8110.

8. Ruijschop RMAJ, Burgering MJM, Jacobs MA, et al. (2009) Retro-nasal aroma release depends on both subject and product differences: a link to food intake regulation? Chem Senses 34, 395-403.

9. Brown WE, Dauchel C \& Wakeling I (1996) Influence of chewing efficiency on texture and flavour perceptions of food. I Texture Stud 27, 433-450.

10. Buettner A, Beer A, Hanning C, et al. (2001) Observation of the swallowing process by application of videofluoroscopy and real-time magnetic resonance imaging - consequences for retro-nasal aroma stimulation. Chem Senses $\mathbf{2 6}$, 1211-1219.

11. Buettner A, Beer A, Hanning C, et al. (2002) Physiological and analytical studies on flavor perception dynamics as induced by eating and swallowing process. Food Qual Prefer 13, 497-504.

12. Wright KM, Sprunt J, Smith AC, et al. (2003) Modeling flavor release from a chewed bolus in the mouth. Part 1. Mastication. Int J Food Sci Technol 38, 351-360.

13. Pionnier E, Chabanet C, Mioche L, et al. (2004) 1. In vivo aroma release during eating of a model cheese: relationships with oral parameters. J Agric Food Chem 52, 557-564.

14. Zijlstra N, Mars M, Stafleu A, et al. (2008) Effect of bite size and oral processing time of food on satiation. BFDG Abstracts. Appetite 51, 753.

15. Zijlstra N, de Wijk RA, Mars M, et al. (2009) Effect of bite size and oral processing time of a semisolid food on satiation. Am J Clin Nutr 90, 269-275.

16. Zijlstra N, Mars M, de Wijk RA, et al. (2008) The effect of viscosity on ad libitum food intake. Int J Obes 32, 676-683.

17. Hetherington M, Rolls BJ \& Burley VJ (1989) The time course of sensory-specific satiety. Appetite 12, 57-68.

18. Hetherington MM \& Boyland E (2007) Short-term effects of chewing gum on snack intake and appetite. Appetite $\mathbf{4 8}$, 397-401.

19. Ruijschop RMAJ (2009) Retro-nasal aroma release and satiation. PhD Thesis, ISBN 978-90-9024360-3. http://arno. unimaas.nl/show.cgi? did=20837

20. Ruijschop RMAJ, Boelrijk AEM, de Ru JA, et al. (2008) Effects of retro-nasal aroma release on satiation. BrJ Nutr 99, 1140-1148.

21. Ruijschop RMAJ, Boelrijk AEM, de Graaf J, et al. (2009) Retro-nasal aroma release and satiation: a review. J Agric Food Chem 57, 9888-9897.

22. Flavor Database by Leffingwell Associates (2004) Retrieved July 31, 2008 http://www.leffingwell.com

23. van Ruth SM, Roozen JP \& Cozijnsen JL (1996) Comparison of dynamic headspace in mouth model systems for flavour release from rehydrated bell pepper cuttings. In Flavour Release in the Mouth. Trends in Food Science and Technology, vol. 7, pp. 444-448 [H van Maarse, AJ Taylor and RST Linforth, editors]. Amsterdam: Elsevier Science B.V.

24. Taylor AJ, Linforth RST, Harvey BA, et al. (2000) Atmospheric pressure chemical ionization mass spectrometry for in vivo analysis of volatile flavour release. Food Chem 71, 327-338.

25. der Heij DG (editor) (1994) Trends in Flavour Research, pp. 59-64. Amsterdam: Elsevier Science.

26. Weel KGC, Boelrijk AEM, Burger JJ, et al. (2003) A protocol for measurement of in vivo aroma release from beverages. J Food Sci 68, 1123-1128.

27. Weel KGC, Boelrijk AEM, Burger JJ, et al. (2004) New device to stimulate swallowing and in vivo aroma release in the throat from liquid and semi-liquid food systems. J Agric Food Chem 52, 6564-6571.

28. Weel KGC, Boelrijk AEM, Alting AC, et al. (2002) Flavor release and perception of flavored whey protein gels: perception is determined by texture rather than by release. J Agric Food Chem 50, 5149-5155.

29. Buettner A \& Schieberle P (2000) Exhaled odorant measurement $(\mathrm{EXOM})$ - a new approach to quantify the degree of in-mouth release of food aroma compounds. Lebensm Wiss Technol 33, 553-559.

30. Land DG (1994) Perspectives on the effects of interactions on flavor perception: an overview. In Flavor-Food Interactions, pp. 2-11 [RJ McGorrin and J Leland, editors]. ACS Symposium Series 633. Washington, DC: ACS.

31. Wansink B (2006) Mindless Eating - Why We Eat More Than We Think. New York: Bantam-Dell. 